\title{
Lingua italiana e femminile
}

\author{
Anna Laura Lepschy, Giulio Lepschy, Helena Sanson \\ University College London
}

\begin{abstract}
Questo articolo esamina alcuni contributi recenti sul sessismo linguistico, con riferimento specificamente all'italiano. Si toccano questioni di carattere teorico e ideologico (compresa quella del "politicamente corretto») oltre ad esempi di natura grammaticale e lessicale: in particolare le parole che designano attività professionali esercitate da donne, e l'uso del suffisso «-essa» nei termini «dottoressa», "professoressa», "studentessa».
\end{abstract}

Parole chiave: lingua italiana, sessismo linguistico, questioni ideologiche, esempi lessicali.

\section{Abstract}

This paper examines some recent discussions on linguistic sexism, with special reference to Italian. It touches on some general theoretical and ideological points (including the question of "political correctness»), as well as specific examples concerning Italian grammar and vocabulary: in particular the designations of female professional activities, and the use of the suffix «-essa» in the words "dottoressa», "professoressa», «studentessa».

Key words: italian language, linguistic sexism, ideological points, lexical examples.

Una quindicina d'anni fa uno di noi dedicò un articolo ${ }^{1}$ ad alcune questioni relative al sessismo linguistico (o al linguaggio sessista) dal punto di vista dell'italiano. L'articolo offriva un esame critico delle Raccomandazioni, da poco pubblicate, di Alma Sabatini. ${ }^{2}$ Vorremmo tornare ora, a quindici anni di distan-

1. «Sexism and the Italian Language», The Italianist, 7, 1987, p. 158-169; in versione ampliata "Language and Sexism», in Zygmunt G. BARANSKI, Shirley W. VINALl (a cura di), Women and Italy. Essays on Gender, London e Basingstoke: Macmillan, 1991, p. 117-138; in traduzione italiana di Miriam VOGHERA, "Lingua e sessismo», L'Italia dialettale, 51, 1988, p. 7-37, e in Giulio LePSCHY, Nuovi saggi di linguistica italiana, Bologna: il Mulino, 1989, p. 61-81.

2. Alma SABATINI, Raccomandazioni per un uso non sessista della lingua italiana. Per la scuola e per l'editoria scolastica (Commissione nazionale per la realizzazione della parità tra uomo e donna), Roma: Presidenza del Consiglio dei ministri. Direzione generale delle informa- 
$\mathrm{za}$, su alcuni punti toccati allora, ed aggiungere qualche riflessione. Il panorama è certamente cambiato, ma non tanto quanto ci si sarebbe potuti aspettare, e non necessariamente in senso positivo, né riguardo all'impostazione teorica generale, né alle indagini relative agli aspetti pertinenti dell'uso italiano attuale, né infine riguardo al peso e alla dinamica del sessismo linguistico in Italia.

Per la situazione teorica generale, considerando come vengono visti i rapporti fra il linguaggio e la mente, e gli aspetti entro i quali il linguaggio determina il nostro modo di pensare, di vedere il mondo, e insomma di percepire la realtà, si è venuto sempre più diffondendo l'assunto secondo cui noi non parleremmo la nostra lingua, ma saremmo da essa parlati, e siccome la lingua, anzi, ogni lingua sarebbe intrinsecamente patriarcale e sessista, tale sarebbe inevitabilmente il nostro modo di pensare (tanto di donne quanto di uomini), e tutta la nostra discorsività. La cosa migliore che potremmo fare è rendercene conto, ed esibire apertamente la natura ideologica di ciò che diciamo, decostruire le nostre narrative illustrandone l'inattendibilità e la contraddittorietà.

Questi atteggiamenti possono essere salutari in quanto provocano una maggiore autocoscienza e ci stimolano a chiarire i presupposti da cui partiamo e le implicazioni del nostro modo di ragionare. Possono anche provocare qualche perplessità quando cercano di smascherare non solo la falsa coscienza, ma ogni coscienza, e di rifiutare qualsiasi riflessione possa essere riportata a tradizionali criteri di verità, scientificità, prova, verifica, ecc. Un'opera storica sarebbe in realtà una narrativa, che va interpretata con gli strumenti offerti dalla retorica. I testi non andrebbero assoggettati a un'analisi storica, che ne stabilisca attendibilmente il significato e la verità. Tutto questo sarebbe in realtà inattingibile, o meglio dipenderebbe dalla creatività e inventività dell'interprete, che non deve essere vincolato da presunti criteri obiettivi di carattere filologico o linguistico. Sebbene in generale da queste discussioni non si ricavi, a nostro parere, molto di utile per un approfondimento teorico di questi problemi, dai contributi migliori (e tanto più quanto più colti e intelligenti sono gli autori, e, paradossalmente, contraddicendo i loro stessi postulati iniziali) si apprendono una quantità di fatti nuovi e di idee interessanti.

Accenneremo ad alcuni lavori che abbiamo avuto occasione di vedere negli ultimi anni. In inglese ci sono interventi stimolanti, come quelli di Robin Lakoff, che ha fatto seguire al suo importante saggio del $1975^{3}$ un volume

zioni della editoria e della proprietà letteraria artistica e scientifica, 1986; anche in Alma SABATINI, con la collaborazione di Marcella MARIANI e la partecipazione alla ricerca di Edda Billi, Alda SANTANGelo, Il sessismo nella lingua italiana, ivi, 1987.

3. Robin LAKOFF, Language and Woman's Place, New York: Harper \& Row, 1975. 
recente, ideologicamente impegnato, il cui taglio post-moderno non offre a nostro avviso l'occasione per un progresso teorico, ma discute utilmente molti episodi relativi al "politicamente corretto» e al sessimo linguistico, soprattutto nella prospettiva della cultura statunitense. ${ }^{4}$

Troviamo anche manuali come quello di Anne Pauwels che presenta una trattazione sistematica del sessismo nel linguaggio, dal punto di vista più generale della "pianificazione linguistica», della "riforma del linguaggio", in una prospettiva internazionale che tiene conto degli studi in lingue e tradizioni diverse. ${ }^{5}$ Dopo due capitoli introduttivi, il volume affronta, in maniera didatticamente ordinata, i seguenti temi: "Should sexist language be changed?», "How should sexist language be changed?», «Implementing nonsexist language change: guidelines», "Evaluating feminist language planning», «Is change occurring?», e alla fine aggiunge un'appendice «Drafting non-sexist language guidelines», e un'utile bibliografia di oltre venti pagine.

In inglese abbondano anche i manuali per la preparazione di manoscritti secondo criteri non sessisti (a volte le norme relative vengono inserite anche nelle istruzioni redazionali generali preparate dalle case editrici), per esempio quelli di Miller e Swift ${ }^{6}$ e di Doyle.

Per l'italiano, dopo le proposte di Alma Sabatini, desideriamo ricordare gli atti del convegno di Sappada Donna \& linguaggio, ${ }^{8}$ e poi la vivace raccolta di articoli ${ }^{9}$ a cura del Progetto Polite, ${ }^{10}$ fra i quali segnaliamo l'intervento di Cecilia Robustelli, particolarmente pertinente per il nostro argomento. ${ }^{11}$ Riprenderemo con qualche aggiunta e nuove riflessioni alcuni dei punti trattati nell'articolo del 1987 citato nella nota 1 .

4. Robin Tolmach LaKoff, The Language War, Berkeley \& Los Angeles: University of California Press, 2000.

5. Anne PAUWELS, Women Changing Language, London: Longman, 1998.

6. Casey Miller, Kate SWIFT, The Handbook of Non-Sexist Writing for Writers, Editors and Speakers, edizione britannica a cura di Stephanie DowrICK, London: The Women's Press, 1981 (seconda edizione britannica a cura di Lesley LEVENE, 1989; terza edizione britannica a cura di Kate Moss, 1995).

7. Margaret DoYle, The A-Z of Non-Sexist Language, London: The Women's Press, 1995.

8. Gianna Marcato (a cura di), Donna \& linguaggio. Atti del Convegno Internazionale di Studi (Sappada/Plodn [Belluno], 26-30 giugno 1995), Padova: Cleup, 1995. Si veda la recensione di Chiara CIRILlo, Lingua e stile, 3 (4), 1998, p. 749-752.

9. Saperi e libertà. Maschile e femminile nei libri, nella scuola, nella vita, a cura di Ethel PORZIO Serravalle, Roma: Presidenza del Consiglio dei Ministri, Dipartimento per le Pari Opportunità; Milano: Associazione Italiana Editori, Cisem, Poliedra Progetti Integrati, 2000.

10. Sul verso del frontispizio, p. 4, si legge: «Il volume è stato realizzato nell'ambito di PolitePari opportunità e libri di testo, progetto cofinanziato dalla Commissone europea nell'ambito del IV Programma d'azione comunitaria a medio termine per le pari opportunità per le donne e gli uomini».

11. "Lingua e identità di genere», in Saperi e libertà, cit., p. 53-68. 
Una questione su cui non si sono avuti progressi o cambiamenti visibili è quella della concordanza grammaticale di aggettivi e participi, secondo il genere (maschile o femminile) del sostantivo a cui si riferiscono (la guardia robusta; la sentinella è stata vistalaggredita) o secondo il genere naturale (sono stata vista; ci siamo rivolte a loro, se parlano donne; sono stato visto; ci siamo rivolti a loro, se parlano uomini; ti sei ribellata; vi siete stupite, parlando a donne; $t i$ sei ribellato; vi siete stupiti, parlando a uomini).

Qui emergono problemi particolari, quando il genere grammaticale delle persone coinvolte è diverso (Mario e Anna si sono baciate/i), con la consueta tendenza a fare prevalere il maschile - che sarebbe un maschile non marcato, secondo alcuni linguisti, un maschile maschilista secondo altri.

Un altro aspetto riguarda la parità di trattamento, cioè il parallelismo nel modo di riferirsi a donne e a uomini. Una questione complessa, e controversa, è quella dell'uso o dell'omissione dell'articolo davanti al cognome. Nell'italiano, parlato e scritto, che noi abbiamo appreso come prima lingua, fra il quarto e l'ottavo decennio del Novecento, i cognomi di donne richiedevano obbligatoriamente l'articolo. Parlare di Maria Corti, e dire "Corti ha scritto questo" ecc., sarebbe stato impossibile: una sgrammaticatura che non sarebbe neppure venuto in mente di fare. L'unica forma per noi familiare era «la Corti».

Con i cognomi di uomo si poteva invece usare o omettere l'articolo («Segre» o «il Segre») e altrettanto si poteva fare con i prenomi da soli ("Cesare» o «il Cesare»; "Maria» o «la Maria») o accompagnati dal cognome ("Cesare Segre» o «il Cesare Segre»; «Maria Corti» o «la Maria Corti»). Queste possibilità diverse non erano indifferenti e intercambiabili, ma caratterizzate, in maniera sottile e complessa, a seconda delle regioni, e di variabili stilistiche, formali e settoriali. Più tardi, dalla fine degli anni Sessanta, ha cominciato a diffondersi, a quanto pare in ambito femminista, l'uso dei cognomi di donna senza articolo, con lo scopo di praticare una parità di trattamento, appunto di stampo «non sessista» ("Corti scrive...» come «Segre scrive...»). Una studiosa dell'università di Reading ha esaminato di recente questo uso, indicando quanto raro esso sia. ${ }^{12}$ Come capita spesso con fenomeni di questo tipo, non è facile appurare come esattamente stiano le cose. Osservazioni episodiche sembrano indicare che il parlato centro-meridionale è meno resistente di quello settentrionale a questo uso. Anche parlanti torinesi di generazioni diverse ci dicono che in certe situazioni di tipo istituzionale, compagne di scuola o insegnanti donne potevano venire designate, già nei primi decenni del Novecento, col cognome senza articolo.

12. Chiara Cirillo, "Corti” or "la Corti"? Definite article + surnames for women», The Italianist, 18, 1998, p. 272-288. 
Un'area instabile e controversa è quella della scelta di nomi (uguali o diversi, per uomini e donne) per professioni o attività che possano essere esercitate da femmine o da maschi. ${ }^{13}$ In quest'area, sia per termini che abbiano una tradizione abbastanza lunga, sia per quelli recenti o per i neologismi introdotti nei nostri anni, si incontrano due tendenze opposte, per la designazione di donne che svolgono certe funzioni.

La prima tendenza, rappresentata dalle Raccomandazioni di Alma Sabatini, favorisce l'uso sistematico di termini che oppongano il genere grammaticale femminile a quello maschile, attraverso (a) suffissi femminili (principalmente quelli in -trice; quelli in -essa, come vedremo vengono esclusi); (b) la desinenza femmminile in $-a$; (c) la concordanza con articoli, aggettivi e participi femminili, nel caso di nomi in - $e$ in - $a$ grammaticalmente "comuni", cioè morfologicamente invariabili.

Alcuni esempi per questi tre gruppi: nel gruppo (a) troviamo forme che non provocano problemi, corrispondenti ai maschili in -tore, come ambasciatrice, amministratrice, direttrice, ispettrice, promotrice, rettrice, scrittrice, senatri$c e$; nel gruppo (b) troviamo forme in -a corrispondenti a maschili in $-o$, come architetta, avvocata, chirurga, critica, deputata, magistrata, medica, ministra, notaia, prefetta, rabbina, segretaria, sindaca, soldata, e corrispondenti a maschili in -e, come cancelliera, carabiniera, consigliera, finanziera, e, in maniera più problematica, a maschili in -sore, -tore come assessora, questora; infine, nel gruppo (c) troviamo forme "comuni», corrispondenti a maschili morfologicamente identici in -e, come un'agente, una comandante, una caporale, una generale, una giudice, una maggiore, una parlamentare, una preside, una presidente, una studente, una vigile; a queste possiamo aggiungere altre forme presentate come "comuni» anche se la loro morfologia sembra etimologicamente meno adattabile al femminile, come una prete, una sacerdote; una poeta, una profeta; ${ }^{14}$ e una Capo di Stato Maggiore, una caposezione ecc.

La seconda tendenza, che pare avere radici più antiche nel movimento femminista, e, a giudicare impressionisticamente, sembra oggi prevalere, preferisce ricorrere, per designare uomini o donne indifferentemente, al termine che abitualmente serve a indicare chi esercita una data funzione, anche se tale termine è di solito grammaticalmente maschile. Questa tendenza preferisce dunque, alle designazioni "comuni», o esplicitamente femminili, quelle che potremmo chiamare «epicene» (termine che si usa per nomi di animali, come pesce, o aqui-

13. Dominic STEWART, «Forms for Women in Italian», The Italianist, 7, 1987, p. 170-192; Chiara CIRILlO, «Gender and Feminine Agentives in Italian Dictionaries: 1612-1917», in Giulio LePSCHY e Prue SHAW (a cura di), A Linguistic Round-table on Dictionaries and the History of the Language, London: Centre for Italian Studies, University College London, 2000, p. 11-23, e la tesi di PhD che Chiara Cirillo sta completando all'Università di Reading.

14. Si noti che per questi nomi il plurale del maschile è in $-i$, quello del femminile fa difficoltà tanto con $-i$ quanto con $-e$. 
la). ${ }^{15}$ Questa preferenza per le espressioni epicene fu ampiamente discussa sui giornali durante il primo governo Berlusconi, quando la presidenza del Senato fu affidata a Irene Pivetti, che desiderava essere designata come «il Presidente», e non «la Presidente» o «la Presidentessa» del Senato. Secondo questa tendenza una donna sarà dunque (in contrasto con le liste presentate sopra) (a) ambasciatore, ${ }^{16}$ amministratore, direttore ecc.; (b) architetto, avvocato, assessore ecc.; e (c), sempre con la concordanza maschile, un agente, un comandante, un caporale, e un prete, un poeta ecc.

La pratica di questa seconda tendenza inciampa a volte in qualche intoppo sintattico, del tipo «è arrivata/o Maria Corti, il famoso filologo", "Maria Corti, il famoso filologo, è arrivato/a» ecc. La prima tendenza incontra invece difficoltà morfologiche, specialmente per le formazioni femminili corrispondenti a quelle in -sore, -tore per gli uomini. Qui troviamo in particolare due termini, dottoressa e professoressa, nei quali appare consolidato (come del resto nel caso di studentessa) il suffisso -essa, che viene però ostracizzato in tutti i suoi usi, in entrambe le tendenze. Su questo punto desideriamo soffermarci nell'ultima parte di questo articolo. ${ }^{17}$

7

L'uso non sessista della lingua può richiedere la proscrizione di forme considerate criticabili, e la prescrizione al loro posto di forme considerate accettabili. Ciò rientra, come si è visto sopra, nella sfera del "politicamente corretto".

Questa etichetta, a quanto pare di origine statunitense («politically correct»), ha un valore ambivalente. Non sempre è chiaro se definire un'espressione come "politicamente corretta» voglia dire appiopparle una denominazione beffarda e sarcastica, usata per qualificare manifestazioni di prepotenza fanatica e intollerante, da parte di chi vuole imporre al modo di parlare proprio (ma anche, e soprattutto, degli altri) i propri pregiudizi; o se si tratti invece di un'etichetta positiva, che presenta l'uso "politicamente corretto" come un ammirevole ideale che bisognerebbe cercare di mettere in pratica.

A proposito del «politically correct» Margaret Doyle osserva: «The origins of the term continue to be debated - some claim it started out as a label created by the right wing for a movement on American campuses to expand the

15. Dal greco epíkoinos (latino epicoenos) «comune, promiscuo». Nell'uso grammaticale si distingue di solito fra parole "epicene», che non cambiano genere grammaticale, ma possono designare femmine o maschi (come il pesce, maschile, o l'aquila, femminile); e parole "comuni», che possono essere trattate come grammaticalmente femminili o maschili, senza variazioni nella loro morfologia (come un amante e un'amante). A volte si trova però che i due termini ("epiceno» e "comune») si usano l'uno col significato dell'altro.

16. Questo pare sia l'uso preferito del Ministero degli Affari Esteri, che chiama ambasciatore anche la donna che esercita tale funzione, e ambasciatrice la moglie, se c'è, di un ambasciatore maschio.

17. Si veda Anna Laura LePSCHY, Giulio LePSCHY, Helena SANSON, «A proposito di -essa», in corso di stampa. 
traditional curriculum; others that it is a term coined by the left as a self-deprecating description of some of its own party-line attitudes. Today, the label has become a broad brush applied to any effort to reflect our changing society that goes against the status quo. [...] "Political correctness" [...] has become a useful (though wildly misapplied) label for ridiculing an opposing viewpoint». ${ }^{18}$ Casey Miller e Kate Swift, nella prefazione alla terza edizione del loro manuale di scrittura non sessista, notano che l'espressione "political correctness" viene usata come «a derisive label intended to imply that advocates of equality are attempting to restrict freedom of speech and enforce language rules»; ma non si tratta solo di sessismo: "the label "political correctness" is used as well of those who - with varying degrees of sensitivity and success - attempt to redress, through language, some of the negative images our culture affixes to people because of their race, religion, ethnicity, sexual orientation, age, physical disability or some other condition that separates them from the mainstream.» ${ }^{19}$

Un intero capitolo, il terzo, nel volume di Robin Lakoff, è dedicato alla "correttezza politica» e sottolinea l'ambiguità della designazione. A quanto pare essa è nata in ambito politicamente di sinistra, per indicare l'adesione alla «linea del partito», e ha poi acquistato, sempre negli stessi ambienti, un uso ironico con riferimento a un eccesso di ortodossia partitica. Ben presto l'etichetta è stata adottata dalla destra per criticare ogni posizione percepita come radicale e libertaria. Il termine, usato polemicamente con grande frequenza all'inizio degli anni Novanta, sembra che stia ora uscendo di moda. Questa etichetta, pur essendo spesso rivolta a stigmatizzare espressioni di fatto sgradevoli, è usata soprattutto per esprimere posizioni illiberali, come indica Robin Lakoff: " "Political correctness", "politically correct", and the common abbreviation for both, "p.c.", cover a broad spectrum of new ways of using and seeing language and its products, all of which share one property: they are forms of language devised by and for, and to represent the worldview and experience of, groups formerly without the power to create language, make interpretations, or control meaning. Therein lies their terror and hatefulness to those who formerly possessed these rights unilaterally, who gave p.c. its current meaning and made it endemic in our conversation». ${ }^{20}$

Prima di definire un'espressione "politicamente corretta» (dandone una valutazione, positiva o negativa), parrebbe opportuno, ovviamente, essere in grado di stabilirne esattamente l'interpretazione, appurando che valore ha per chi la usa, e per chi è designato (e può sentirsi compiaciuto o irritato) da tale espressione. La questione ricorda i dibattiti recenti sulle proposte secondo cui per denunciare un reato di molestia sessuale, o di razzismo, occorre basarsi sulla reazione non (come è ovvio) di chi commette il reato, né di un osservatore esterno e (si presume) obiettivo, bensì di chi è l'oggetto delle molestie o del razzismo, e documenta in prima persona, proprio nella sua qualità di vittima, 
nel suo sentirsene offeso, l'avvenuto reato. Criterio per certi aspetti ammirevole, ma anche, come è evidente, difficile da applicare in maniera equa. La questione è resa anche più complessa dal fatto che un gruppo subalterno può rivendicare per sé con fierezza un termine prima usato con valore spregiativo da chi detiene il potere di imporre lo standard: si pensi al caso di negro in America prima considerato normale, e poi sostituito da black, in origine insultante, in seguito adottato con orgoglio («black is beautiful»), e al quale, dopo che il termine è diventato normale, viene ora spesso preferito African American. In altre lingue la situazione è inevitabilmente diversa, e i rapporti fra negro e nero in italiano non sono gli stessi. Per un'ampia, pensosa e informata rassegna di parole che appartengono alla sfera dell'alterità, delle designazioni del "diverso", si veda il recente volume di Federico Faloppa. ${ }^{21}$

Similmente, chi decide se un termine è offensivo, e come tale meritevole di ostracismo? Prima di passare ai tre termini dottoressa, professoressa e studentessa, prendiamo un caso interessante, quello della parola inglese Jewess.

Questo termine si può trovare usato in maniera neutra, senza connotazioni negative. Ma leggendo i testi sul linguaggio sessista si ha un'impressione diversa. Per esempio, in Miller e Swift si legge: "Attached to proper nouns, -ess endings are especially offensive. Fortunately Negress, Jewess, Quakeress, etc., are almost defunct today». ${ }^{22}$ E nella guida di Margaret Doyle, a proposito delle forme in -ess si osserva che alcune sono più deplorevoli di altre: "On the "reasonable" end of the scale, for example, may be found actress; at the opposite extreme may be terms that are both sexist and racist such as Jewess and Negressi. ${ }^{23}$ Questi giudizi potrebbero forse essere attribuiti a una smodata "correttezza politica", ma sembrerebbe azzardato attribuire lo stesso eccesso ai vocabolari inglesi usuali che si trovano sugli scaffali di consultazione della British Library, in cui si leggono, non come alternative, bensì come unico valore della voce Jewess, le seguenti definizioni: "offens. a female Jew»; ${ }^{24}$ "an offensive term referring to a Jewish woman or girl (dated offensive)»; 25 "dated or offensive a Jewish woman or girl»; ${ }^{26}$ fem (offensive when used by non-Jews)». ${ }^{27}$

Di fronte a questa lista di giudizi perentori si resta perplessi. Conviene considerare la posssibilità che i vocabolari sbaglino, nel registrare il termine come insultante, trascinati dal desiderio di essere più realisti del re, cioè ancora più «corretti» dei sostenitori della "correttezza politica». La scelta della parola Jewess avrebbe allora un valore politico, ma non quello di servirsi di un termine insultante, bensì quello di rivendicare il diritto di usare un termine che insultante

21. Federico FALOPPA, Lessico e alterità. La formulazione del "diverso», Alessandria: Edizioni dell'Orso, 2000.

22. Miller e SWIFT, The Handbook, cit., p.138.

23. DoYLe, The $A-Z$, cit., p. 27.

24. The Concise Oxford Dictionary of Current English, Oxford: Clarendon Press, 1998 (ristampa della nona edizione del 1995).

25. Encarta World English Dictionary, London: Bloomsbury, 1999.

26. The New Penguin English Dictionary, London: Penguin Books, 2000.

27. The Chambers Dictionary, Edinburgh: Chambers, 1998 (s.v. Jew). 
non è, né lo diventa solo perché ci sono dei vocabolari che così lo qualificano. Questo termine, come tanti altri, potrà avere un valore offensivo, ma può anche essere usato in modo neutro. Servendosene in un contesto insospettabile si intende forse rivendicare la libertà di usare il linguaggio tradizionale senza lasciarsi intimidire da chi vuole espungere dall'uso comune espressioni che trova ideologicamente sgradite. Questo può indurre a riflettere sulla difficoltà di attribuire con tanta sicurezza e intransigenza valori politici e ideologici alle parole che si usano (soprattutto a quelle che usano gli altri). Si tratta beninteso di una questione di fatto, e non di principio. Bisogna decidere, caso per caso, se certe espressioni siano ineccepibili, discutibili, o inaccettabili, e questo spesso dipende dal momento, dal contesto e dalle circostanze. La scelta del lessicografo non è facile, ma non è detto che mirare a un massimo di "progressismo" sia sempre la soluzione migliore e più efficace. Tanto più che i vocabolari godono di un'autorità fondata su motivi socio-culturali più che linguistici, e le loro definizioni influenzano l'uso anche se di fatto non lo rappresentano fedelmente. Un termine può essere generalmente considerato insultante più perché il vocabolario lo qualifica così che perché esso venga di fatto adoperato in maniera spregiativa nell'uso comune. Spesso si tratta dunque di valutazioni che contribuiscono a creare la situazione che descrivono.

\section{8}

I tre termini dottoressa, professoressa, studentessa intorno alla metà del Novecento potevano apparire innocui e saldamente stabiliti in italiano. Chi li usasse allora, o ancora li usi oggi tranquillamente e senza remore, può restare sorpreso dalla loro storia.

L'unico dei tre attestato da circa cinque secoli è dottoressa, che però in tutto il corso della sua storia fino ai primi del Novecento, è stato usato in modo prevalentemente negativo e beffardo, per indicare donne saccenti e presuntuose. I vocabolari dell'Ottocento danno anche la forma dottora (preferito anche nelle Raccomandazioni di Alma Sabatini). Per dottoressa il Fanfani (1855) dà la definizione «Donna sacciuta, e salamistra", e per dottora "Dottoressa, Salamistra, e dicesi di Donna che vuol far la saputa e metter la bocca in quel che non le tocca». ${ }^{28}$ Il Rigutini-Fanfani (1875) dà per dottoressa "Donna che vuol far la saputa, Che vuol parer dotta: "Si cheti lei, dottoressa: — La signora Lucrezia è una gran dottoressa, e vuol parere di intendersi di tutto" »; e per dottora «Lo stesso che Dottoressa, e dicesi di donna che vuol far la saputa e metter bocca da per tutto: "Si cheti lei, dottora: - Vuol far sempre la dottora" ${ }^{29}$ Il Tommaseo-Bellini (1865-1879) osserva che dottora non "ha il senso veramente di Donna addottorata», e dà l'esempio far la dottora: "Voler parere saputa, o savia, Dar sentenze e consigli». Anche dottoressa, che viene presentato come più usua-

28. Pietro Fanfani, Vocabolario della lingua italiana, Firenze: Le Monnier, 1855.

29. Giuseppe Rigutini, Pietro FANFANI, Vocabolario italiano della lingua parlata, Firenze: Tipografia Cenniniana, 1875 . 
le, «di donna addottorata, sul serio, non è com.; ma suonerebbe men cel. che Dottora. Più conveniente dirla Addottorata». Naturalmente non manca il significato di «Donna che vuole sdottorare. Più com. che Dottora; e dicesi tanto delle letterate, quanto delle sputasentenze anco nelle cose di casa». ${ }^{30} \mathrm{E}$ ancora nel 1905 il Panzini, alla voce dottora, commenta: «femminile di dottore e meno comune di dottoressa. Ora le donne addottorate in qualche disciplina, così fiere come esse oggi sono della loro dignità, come chiamarle? a dottora non ci si ausa e dottoressa sa di saccente, e pare contenere in sè alcuna parte di scherno o almeno di estraneo all'ideale femminista: onde è che le donne che hanno diploma di laurea, scrivono spesso sul biglietto dottore, quasi nome partecipante. La grammatica del Morandi e Cappuccini (\$138) approva questo nuovo uso femminile di dottore. Così in fr., fermme docteun.${ }^{31}$ La sesta edizione, del 1931, aggiunge: «Anche una poetessa oggi è poeta. Non bastano i maschi?». Nell'ottava edizione postuma, del 1942, si legge solo che dottora è il femminile di dottore, meno comune di dottoressa. Quest' ultimo si era evidentemente del tutto affermato.

Professoressa secondo i vocabolari è attestato dal $1897,{ }^{32}$ e studentessa dal 1907. I vocabolari ottocenteschi danno professora, ma non professoressa. Il Rigutini-Fanfani (1880) alla voce professora annota: «femm. di Professore; ma si userebbe più spesso per ischerzo: "Vuol far la professora, ma non sa nulla"».33 Il termine studentessa manca nei vocabolari ottocenteschi; quelli che danno studente a volte indicano che si tratta di un sostantivo maschile, a volte non specificano il genere grammaticale e lasciano aperta la possibilità di considerarlo "comune» (lo studente, la studente). Migliorini cita un passo in cui Carducci nel 1891 scrive «le signorine studenti». ${ }^{34}$ Ancora nel 1926 in un romanzo di Liala si trova un liceale che viene corretto dal suo professore: «E lei non dica studentesse $[. .$.$] Si dice [. .$.$] studenti». { }^{35}$

L'affermazione di dottoressa, professoressa, studentessa nel Novecento sarà presumibilmente dovuta all'uso ufficiale nell'ambito della pubblica istruzione. Ė lecito chiedersi se questi tre termini sopravviveranno ancora indisturbati, o se la loro disponibilità si avvicini alla fine, dopo meno di un secolo di impiego relativamente pacifico.

30. Nicolò Tommaseo, Bernardo Bellini, Dizionario della lingua italiana, Torino: Unione tipografico-editrice, 1865-1879.

31. Alfredo PAnZInI, Dizionario moderno. Supplemento ai Dizionari italiani, Milano: Hoepli, 1905.

32. Ma il Fornaciari usa il termine, sia pure dubitativamente, nel 1881: «da professore si farebbe professoressa», Raffaello FORNACIARI, Sintassi italiana dell'uso moderno, Firenze: Sansoni, 1881, p. 19.

33. Giuseppe Rigutini, Pietro FANFANI, Vocabolario italiano della lingua parlata, terza impressione sulla edizione emendata, Firenze: Barbera, 1880 (la voce manca nella prima edizione del 1875).

34. Bruno Migliorini, Storia della lingua italiana, Firenze: Sansoni, 1960, p. 713.

35. Liala, Ombre di fiori sul mio cammino, Milano: Sonzogno, 1997, p. 8 (il romanzo, pubblicato nel 1981, pare risalga al 1926). 